\title{
Comparative Study on Change Management
}

\author{
Tariq Rahim Soomro \\ College of Eng. \& IT \\ Al Ain University of Science \& Technology, Al Ain,
} UAE

\author{
Amanullah Bashir \\ Department of IT \\ SZABIST, Dubai Campus \\ United Arab Emirates (UAE)
}

\begin{abstract}
Change is vital in all types of organizations. The main focus of change is to always improve the business; and it must be able to execute change with least cost and least risk of business disruption. The study mainly focuses on change management process of Information Technology Infrastructure Library (ITIL) as there are several tools from different vendors, which support ITIL. This study will explore the similarities and differences among the prominent tools and produce comparative study on change management.
\end{abstract}

\section{General Terms}

ITIL, Change Management.

\section{Keywords}

Change Management, BMC, CA.

\section{INTRODUCTION}

The term Change is defined as "process of moving from one defined state to another". Change is a critical aspect of every business. The main role of the changers is to help businesses to run continuously in the consistence state and the Information Technology (IT) organizations must be capable of effectively handling change. IT must also be able to keep its infrastructure and services well-aligned with changing business goals and priorities. In today's fast-moving market, the ability to easily and appropriately handle change is even more important than before; that is why IT organizations need to implement and automate best practices for the entire endto-end Change Management lifecycle. Only those IT organizations that embrace this disciplined approach to change management will be able to deliver the operational agility essential for service excellence [1][2]. This paper highlights the comparative study on Change Management process based on different vendor's tool. Each vendor has different approach to implement the change management process based on Information Technology Infrastructure Library (ITIL) change management process [3]. The next section will discuss why change management is important for organizations; Latter the paper will focus on the similarities and differences between the prominent tools of ITIL form two different vendors and finally in discussion section we will conclude the paper.

\section{WHY CHANGE MANAGEMENT?}

Information technology is an integral part of all organizations and it is becoming more critical in business operations. The business strategies, the services and technologies are changing at high rate. The users are requiring best level of services to meet challenging business objectives. All these factors are collectively require an IT environment where changes are controlled and managed at high preciseness. The main objective of the Change management process is to ensure change's record, evaluation, authorization, prioritization, planning, testing, implementation, and reviewing in a controlled manner [4].

\section{PROMINENT TOOLS}

Currently organizations are using several different tools from different venders to implement their best practices, among them there are two prominent tools.

1. BMC Software: BMC Inc. offers a comprehensive approach and unified platform that helps IT organizations cut cost, reduce risk and drive business profit. The BMC Software was founded in September 1980. BMC Software have the brands; Atrium, main View, BEST/1, Marimba, BladeLogic, MQSoftware, Control-M, PATROL, Control-SA, ProactiveNet, Remedy, and Magic [5].

2. Computer Associates: CA Technologies, formerly CA, Inc. and Computer Associates, Inc., is one of the largest independent software corporations in the world. In 2010, CA Technologies announced its cloud computing management strategy at its CA World user conference, and its new CA Cloud-Connected Management Suite of products that address the emerging challenges presented by the cloud [6].

The comparison between BMC Change management process and CA Change management Process is discusses as follows:

BMC Change Management Process has eight procedures, six for implementing planned changes, and two for emergency changes.

\subsection{BMC Procedure: Request for Change Review}

The change request can start from the Incident Management, Problem Management, Continuity Management and Release Management. If user requirements are already there in the prepared change then add request for change to existing change otherwise request for standard change and then prepare change using appropriate template and if change request has conflict with internal policies or standards reject the change request otherwise request has been passed to change management. If Release Management is required then pass request for change to Release Management otherwise prepare change using template [7][8][9]. CA Change Management Process has eight activities.

\subsection{CA Activity: Request for change}

Change Management starts with a Request for Change (RFC). RFC could be initiated by a customer request via the Service Desk, the introduction or removal of a Configuration Item (CI), or development project output. It can be initiated by 
Problem Management. To prevent a situation of many entry points into the change process, documentation about owner of RFC creation, what those RFCs are intended to do? And what information is required by them? A more suitable approach would be to use service requests for routine standardized demands that need not to be controlled by Change Management and to use IT or business relationship managers to submit RFCs. The main objective of Change Management is to facilitate all change requests using clear procedures, automated simple checks and balances. Each and every member of the organization should have rights to submit an RFC [1][8][10].

\subsection{BMC Procedure: Change Planning}

When a newly change entry is enrolled, risk and impact analysis phase starts by collecting important related information so that possibility of creation of change implementation plan with minimal failure risk and impact on user occur. In case of build a new service infrastructure or amendment in an existing one in order to satisfy change requirements. After getting risk and impact information, finalization for the change implementation plan ensure risk of failure and impact on user is minimized. Approval phase can be skipped in case of standard change; the change supervisor initiates the change implementation. If approval is required the change supervisor asks the change manager to start the collection of the important approvals [7][8][9].

\subsection{CA Activity: RFC Analysis}

Analysis is very critical for any type of change. An initial evaluation of the information provided for completeness and feasibility is performed in RFC analysis phase. An automated system can significantly shorten this phase by allowing the tool to apply business rules to determine what information is required. A key requirement for such a system is the ability to ensure that adequate change lead times are in place, and are in line with policy [1][10].

\subsection{CA Activity: Change Prioritization}

Change prioritization is done after the initial RFC analysis. This occurs by analyzing the impact of incident, problem, project or organization's importance of whatever change is implementing. The Change Advisory Board (CAB) may intervene if it is not agreed. When the change priority is fixed, it is used to find resource requirements and change scheduling windows. In a resource-constrained environment, business units can use it to internally prioritize demand. Risk assessment should also be finding at this stage. Risk is always involved when implementing a new change. Measuring risk can be defined as the actual risk of change implementation versus the risk of possible failures if changes are not implemented. Both types of risks cannot be ignore and should be evaluated and cost-analyzed. Impact of the change is another aspect of risk determination. The impact analysis has a crucial role in Change Management process. Today in a highly shared environment, an individual cannot track all of the touch points between technology and business services. What is the impact on Service Level Agreements (SLAs)? And what business services are affected? Is also very critical. Integration of Change Management with Configuration Management can help to determine the impact of a technology change on business services [8][10].

\subsection{CA Activity: Build and test change}

The Categorization activity is a major hub for a number of activities. Categorization consists of change size evaluation from a resource requirements perspective, the risk, priority, and then deciding what process steps you will follow. Categorization is an extremely important activity, since it is assigned according to business impact, and therefore determines the level of change authorizations and financial and resource requirements. During this stage, IT must collaborate with the business to ensure the correct categorization of changes and to avoid problems further go down in the line. The most work of Change Management is done at this stage, with many checks and balances to ensure that change approval becomes straightforward. Here, realization of the major benefits of Change Management by utilizing technologies to help determine change categories (based on criteria agreed upon with the business), to quickly absorb large volumes of changes, and to determine the costs of changes before they are incurred [1][8].

\subsection{CA Activity: Categorize}

The change testing is very important and is done on test environment. Testing helps to refine the change implementation, any error or bug can be found during the testing. Some hidden behaviors of change can also be found during testing and more chances of success increases. Implementation of change on production without testing is always a big risk and implementer should discourage for it. Change implementation on production without testing has the high risk of change failure [1][10].

\subsection{BMC Procedure: Change Approval}

Review is done by Change Manager; if he/she finds conflict with internal policies or standards then it is informed that change cannot be implemented. In case of no conflict with internal policies, change assessment and implementation reviews by change manager. The change manager checks the plan to ensure that user is not affected by the impact of the change and there is no failure of risk is involved. One more consideration is implementation time, which would not be conflict with other planned events and changes. On the one hand if change planning has some gaps which would be cause for the failure; or planning conflicts with other events or planned changes, then change manager asks change supervisor for the adjustment of the implementation plan. On the other hand, if change planning implementation does not have any impact and no failure of risk is involved, the change manager ensures approvals for the change from the customers who would be affected by the change. In case of the change is not approved by an approver, the change manager digs out the reason for the rejected change and he/she ask to change supervisor for additional risk analysis. If change was rejected due to some other reasons then change manager informs change supervisor that possibility for the change implantation is not there [7][8][9].

\subsection{CA Activity: Change Advisory Board Review}

After Categorization activity, there is Change Advisory Board (CAB) Review meeting is must for a decision of change, weather to go for change or not. The $\mathrm{CAB}$ consists of all the interested parties for active changes, both from IT and the business. The $\mathrm{CAB}$ should follow meeting procedures, including meeting minutes and communication. The $\mathrm{CAB}$ should review all proposed and implemented changes. For new changes, there should be agreement on the need, resource allocation and available funds. The business must be involved from the design phase of process development. To determine the quality of the process the $\mathrm{CAB}$ should also review implemented changes and whether the changes were implemented correctly. Determining that the technical aspect 
of the change was successfully implemented is not enough, it is required is to determine change achieved its goal. More mature organizations will wait for a specified period before closing a change. The $\mathrm{CAB}$ is responsible for approving or rejecting changes. In case of incomplete information, they will send the change back to the requester. Approval must be gained at three levels: technical, business and financial. Once the change is approved by $\mathrm{CAB}$, the phases of scheduling, building, and testing and implementation starts. The key stakeholders should work out scheduling to ensure that all the implementation steps required to institute the change are achievable. Providing visibility into when changes are to be implemented is critical. It should clearly state if any business services or technologies will be impacted, along with the start and end time of the implementation. The $\mathrm{CAB}$ should consider change conflicts or situations where the time of the planned change could have a detrimental effect on the business. The Service Desk aware of planned change and service outages. Otherwise, the Service Desk might waste time trying to diagnose the increase in incidents caused by the implemented change [1][8].

\subsection{BMC Procedure: Infrastructure Change Implementation}

If the change concerns an application change, the implementation continues in Application Change Implementation. The first task of an infrastructure change is assignment of the change to the specialist for the implementation preparation. This can involve configuration of a test environment and performing tests. When everything is ready, the specialist receives and completes the task for the actual implementation of the change [7][8][9].

\subsection{BMC Procedure: Application Change Implementation}

When first task of implementation of the application has been assigned, the insurance of change requirements is tested by the specialist, who will check that new release is fulfilling the user requirements or not. This would be done in test environment if one has made available for this; otherwise test would perform in the development environment. In case of any error occur during testing, the specialist will fix it. The specialist will also update the scripts for regression testing. When it is confirmed by specialist that change requirements have been met and no errors have been introduced, the release administrator transfers the new release to test environment where testing is ensured by the customer. In case of customer had not passed testing, the change supervisor creates and assigns additional tasks, so that new release can be corrected. The approval for change again required if additional tasks caused the transfer to production is rescheduled. Once customer has passed testing for new release, the specialist ensures everything is ready to take new release into production. New release transfers to production by release administrator [7][8][9].

\subsection{CA Activity: Implementation}

The implementation starts after CAB approval. Only approved changes can be implemented. There is no chance for people to introduce unauthorized changes. The entire idea of the Change Management process is to protect the production environment. The implementation report will be reviewed at the next $\mathrm{CAB}$ meeting to ensure that the business goals were met and the risks and costs to the business were minimized [1][8][10].

\subsection{BMC Procedure: Planned Change Closure}

After change implementation into production, verification of successful change implementation would be done by a specialist with the help of a user. If user is not satisfied with the implementation of change or there is no improvement in the system or security risk involves, then change would be backed out. The change supervisor assigns new additional tasks to improve change implementation. The change supervisor finally ensures that the requester and approvers of the change are informed. The requester then checks that requirements are completed and there is no need for further action. If requester's requirements are not completed then he/she can reopens the change [7][8][9].

\subsection{BMC Procedure: Emergency Change Implementation}

Emergency has no time, any time it can happen, so emergency change implementation exists in BMC change management process. In case of incident resolution in emergency situation, emergency change implantation comes into picture. If emergency change concerns an infrastructure change, the implementation would be done as normal incident request. Alternatively, if emergency change is an application change, then creates a new release in development environment to fix incident without any error or bug. The new releases are tested in test environment and if it fails then again work in development environment to rectify the error or bug. In case of release passed tests then implement the change on production environment [7][9].

\subsection{BMC Procedure: Emergency Change Closure}

On production environment testing of change starts after completion of implementation. In case of implementation turns out to be unsuccessful, the situation would be discussed in detail. Conversely, if implementation is successful then complete the incident request. In addition a change is registered in the service management application for the emergency change implementation. Before closing the change, change supervisor informs the service provider that the emergency change has been implemented successfully [7][8].

\subsection{CA Activity: Emergency Changes}

In a dynamic operational environment there will often be times when high-impact incidents and problems need to have fixes applied that will involve a change to the production environment. This is not an opportunity to bypass the change process as there should also be a process in place to handle urgent or emergency changes. Change approval is still a prerequisite in these cases, but the standard process will generally be condensed and some of the $\mathrm{CAB}$ approvals will be delegated. Organizations that have not fully developed their Change Management processes will see a high volume of emergency changes, most likely because of timing. This is normally due to lead times not being enforced, with changes continuously and mistakenly viewed as emergencies. A good metric is to find is it an emergency change? And if there is a high impact incident or problem open; that this change will fix. If this is not the case, then you must question whether it's an emergency change? [8][10]. 


\section{DISCUSSION AND CONCLUSION}

The main aim of Change management Process is to implement the changes with minimum error and maximum efficiency and maintain level of service without interruption. The success of a change can be measured by comparing the defined objectives against the actual status and characteristics of the related configuration item involved in the change after it has been implemented. The change management process wherever possible should follow the guiding principle minimizing the level of service disruption during the planning and implementation of the change, as well as attempting to ensure that there are no related incidents caused by the change itself and helps to ensure that the business is not negatively impacted by change activity [2]. Effective change management always has a positive impact on Business. It reduces downtime, increases efficiency and helps to create a consistent, repeatable business process. The various elements of a change-management process-risk analysis, conflict analysis, and so forth — can be used to solve specific business problems and to make the environment better able to handle new changes to the business. These processes can also help make the more responsive to business needs so that changes with the potential to positively impact the business environment receive increased attention. Change management also has an impact on the security of your environment. It allows your environment to be more secure and provides hooks for change auditing, a process that is becoming more important to many companies, and even legally mandated for others [7]. To Compare Change Management processes of different vendors which are based on ITIL and find out similarities and differences in the vendor tools was the main objective of this study. Both BMC Software and Computer Associates (CA Inc) have their own processes to implement the Change management, but those processes are based on ITIL processes for the Change management. Each vendor tries to design process of change management in a way that it will align with the business needs and with ITIL processes. Each vendor will include its technical area, management area, history of products and processes to form in Change management process. In this study we had compare change management process of BMC Software and Computer Associates which proved that there are few similarities, which can lead towards standardization of ITIL processes and there are some differences, which causes at least change management not to go towards standardization of ITIL procedures [1] [5]. It is no denying a fact, keeping in view the finding of this study that organizations are dependence on tools rather than their best practices. Some similarities are highlighted as follows:

Request for Change (RFC): this procedure is same in both $\mathrm{BMC}$ and CA. The change request can start from the Incident Management, Problem Management, and Continuity Management in BMC. RFC could be initiated by a customer request via the Service Desk, the introduction or removal of a Configuration Item (CI). It can be initiated by Problem Management. Change Approval: the main theme for change approval activity is same for BMC and CA. The main objective of Change Approval in BMC is to control the change conflicts with internal policies, change assessment, impact of change, and no failure risk. One more consideration is implementation time, which should not be conflict with other planned events and changes. The main aim of $\mathrm{CAB}$ in $\mathrm{CA}$ is to review all proposed and implemented changes to determine the technical aspect of the change; it is required to determine change achieved its goals. It should clearly state if any business services or technologies will be impacted, along with the start and end time of the implementation. The $\mathrm{CAB}$ should consider change conflicts or situations where the time of the planned change could have a detrimental effect on the business. Change Implementation: is also same in both $\mathrm{BMC}$ and CA. Change implementation is done after approval in both BMC and CA. When it is confirmed that change requirements have been met and no errors have been introduced, the change promotes to production environment. In $\mathrm{CA}$, implementation starts after $\mathrm{CAB}$ approval, only approved changes can be implemented. The entire idea of the Change Management process is to protect the production environment; unauthorized changes put this objective at risk. The implementation report will be reviewed at the next $\mathrm{CAB}$ meeting to ensure that the business goals were met and the risks and costs to the business were minimized. Emergency Change: is also same in both BMC and CA. In BMC, in case of incident resolution in emergency situation, emergency change implantation comes into picture. If emergency change concerns an infrastructure change, the implementation would be done as normal incident request. In $\mathrm{CA}$, in a dynamic operational environment there will often be times when highimpact incidents and problems need to have fixes applied that will involve a change to the production environment. This is not an opportunity to bypass the change process as there should also be a process in place to handle urgent or emergency changes [1][7]. Some differences are highlighted as follows:

Change Planning: is only in the BMC change management procedure. When a newly change is enrolled, risk and impact analysis phase starts by collecting important related information so that possibility of creation of change implementation plan with minimal failure risk and impact on user. In case of build a new service infrastructure or amendment in an existing one in order to satisfy change requirements, request help from availability manager for creation of new one or amendment of an existing service infrastructure design. After getting risk and impact information, finalization for the change implementation plan ensure risk of failure and impact on user is minimized. In CA change planning is divided in three sub activities as RFC Analysis, Change Prioritization, and categorize. RFC Analysis: is an initial evaluation of the information provided for completeness and feasibility is performed in RFC Analysis phase. An automated system can significantly shorten this phase by allowing the tool to apply business rules to determine what information is required. A key requirement for such a system is the ability to ensure that adequate change lead times are in place, and are in line with policy. RFC Analysis is the separate activity in CA but it does not exist separately in BMC. Change Prioritization: Change prioritization is done after the initial RFC analysis. This occurs by analyzing the impact of incident, problem, project or organization's importance of whatever change is implementing. The Change Advisory Board (CAB) may intervene if it is not agreed. When the change priority is fixed, it is used to find resource requirements and change scheduling windows. Risk assessment should also be found at this stage. Risk is always involved when implementing a new change. Measuring risk can be defined as the actual risk of change implementation versus the risk of possible failures if changes are not implemented. The impact analysis has a crucial role in Change Management process. Categorize: The Categorization activity is present in CA change management process activities, Categorization consists of change size evaluation from a resource requirements perspective, the risk, priority, and then deciding what process steps you will follow. 
During this stage, IT must collaborate with the business to ensure the correct categorization of changes and to avoid problems go further down. In BMC change management procedure, same thing is done in Change Planning. Build and test change: In CA, Build and test change is a separate activity but in BMC testing is done in Infrastructure Change Implementation procedure and Application Change Implementation procedure. Planned Change Closure: this procedure exists only in BMC but it does not exist in CA change management process activities. Emergency Change Closure: this procedure also exists in BMC but it does not exist in CA change management process activities [1][7].

\section{REFERENCES}

[1] Technology Brief: Change Management, 2011, Change Management: A CA Service Management Process Map, http://www.ca.com/files/technologybriefs/change_mgmt _tb.pdf

[2] Rob Addy, 2007, Effective IT Service Management: To ITIL and Beyond, Springer, $1^{\text {st }}$ Edition, ISBN-10: 3540731970, ISBN-13: 978-3540731979

[3] Soomro, T.R. and H.Y. Wahba, 2011, Role of Information technology infrastructure library in data warehouses. American Journal of Applied Science; 8:1384-1387, DOI: 10.3844/ajassp.2011.1384.1387, http://thescipub.com/abstract/10.3844/ajassp.2011.1384. 1387
[4] EduTech Wiki, 2010, Change Management, http://edutechwiki.unige.ch/en/Change_management

[5] BMC Software Inc., 2011, About BMC Software, http://www.bmc.com/corporate?intcmp=foot_about

[6] CA Inc. 2011, What are 5 questions every customer asks about CA Services? http://www.ca.com/us/ /media/CB2F1966A1204CEC82 5DB1CA22DDFB5F.ashx

[7] BMC Software Inc., 2011, BMC Software version 7.0

[8] Jacques Sauvé, Rodrigo Rebroucas, Antao Moura, Claudio Bartolini, Abdel Boulmakoul, David Trastour, 2006, Business-driven decision support for change management: planning and scheduling of changes, Hewlett-Packard Development Company, L.P., Approved for External Publication, https://www.hpl.hp.com/techreports/2006/HPL-200692.pdf

[9] BMC.com Solutions, 2011, IT Infrastructure Library (ITIL), http://www.bmc.com/solutions/esm-initiative/ITInfrastructure-Library-ITIL.html?cmp=redirect_itil

[10] CA Technologies, 2011, ITIL - Community and Insights, http://www.ca.com/us/itil.aspx 